\title{
Home-based chlamydia and gonorrhoea screening: a systematic review of strategies and outcomes
}

Muhammad S Jamil ${ }^{1 *}$, Jane S Hocking ${ }^{2}$, Heidi M Bauer ${ }^{3}$, Hammad Ali $^{1}$, Handan Wand ${ }^{1}$, Kirsty Smith', Jennifer Walker ${ }^{4}$, Basil Donovan ${ }^{1,5}$, John M Kaldor ${ }^{1}$ and Rebecca J Guy ${ }^{1}$

\begin{abstract}
Background: In many countries, low Chlamydia trachomatis (CT) and Neisseria gonorrhoeae (NG) screening rates among young people in primary-care have encouraged screening programs outside of clinics. Nucleic acid amplification tests (NAATs) make it possible to screen people in homes with self-collected specimens. We systematically reviewed the strategies and outcomes of home-based CT/NG screening programs.

Methods: Electronic databases were searched for home-based CT and/or NG screening studies published since January 2005. Screening information (e.g. target group, recruitment and specimen-collection method) and quantitative outcomes (e.g. number of participants, tests and positivity) were extracted. The screening programs were classified into seven groups on the basis of strategies used.
\end{abstract}

Results: We found 29 eligible papers describing 32 home-based screening programs. In seven outreach programs, people were approached in their homes: a median of $97 \%$ participants provided specimens and $76 \%$ were tested overall (13717 tests). In seven programs, people were invited to receive postal test-kits (PTKs) at their homes: a median of 37\% accepted PTKs, 79\% returned specimens and 19\% were tested (46225 tests). PTKs were sent along with invitation letters in five programs: a median of 33\% returned specimens and 29\% of those invited were tested (15126 tests). PTKs were requested through the internet or phone without invitations in four programs and a median of 32\% returned specimens (2666 tests). Four programs involved study personnel directly inviting people to receive PTKs: a median of $46 \%$ accepted PTKs, $21 \%$ returned specimens and 9.1\% were tested (341 tests). PTKs were picked-up from designated locations in three programs: a total of 6765 kits were picked-up and 1167 (17\%) specimens were returned for screening. Two programs used a combination of above strategies (2395 tests) but the outcomes were not reported separately. The overall median CT positivity was 3.6\% (inter-quartile range: 1.7-7.3\%).

Conclusions: A variety of strategies have been used in home-based CT/NG screening programs. The screening strategies and their feasibility in the local context need to be carefully considered to maximize the effectiveness of home-based screening programs.

Keywords: Sexually transmitted infections, Chlamydia trachomatis, Screening, Home

\footnotetext{
* Correspondence: Mjamil@kirby.unsw.edu.au

${ }^{1}$ The Kirby Institute, University of New South Wales, Sydney, NSW, Australia

Full list of author information is available at the end of the article
} 


\section{Background}

Chlamydia trachomatis (CT) is the most common notifiable sexually transmissible infection (STI) in the United States (US) [1], Europe [2] and Australia [3]. Many countries have experienced substantial increase in reported CT infections over the past decade. Screening for CT and Neisseria gonorrhoeae (NG) is important because most infections remain asymptomatic and often undiagnosed $[4,5]$. Untreated infections can result in major sequelae including pelvic inflammatory disease, ectopic pregnancy, chronic pain, and infertility in women and epididymitis in men [5].

Clinical guidelines recommend annual CT screening for sexually active young women in many countries [6-8], and also sexually active men in some countries [9]. For NG screening, local prevalence and individual risk factors should be considered [6,8]. Opportunistic screening of people attending primary-care clinics for nonsexual health reasons has usually failed to achieve high coverage [10-12]. This may be due to practitioner reported barriers including lack of knowledge of the benefits of screening, concerns about upsetting patients, time constraints, lack of reminder systems and little support for contact tracing $[13,14]$. Low attendance rates for routine care among many at-risk people, particularly young men, also play a role in low screening rates [15-17].

The advent of nucleic acid amplification tests (NAATs) has made it possible to screen people in homes with self-collected specimens. Home-based screening, with urine or self-collected vaginal specimens, has been shown to be acceptable and has the potential to reach people who do not get tested otherwise [18]. A recent review reported that home-based STI screening resulted in up to 11 times higher testing rates compared to the clinic-based screening [19]. A randomized control trial (RCT) showed that $83 \%$ of women in a home-based CT screening arm indicated a preference for future homescreening compared to $49 \%$ in the clinic arm who preferred future screening in clinics [20]. We conducted a systematic review of published literature on home-based CT and NG screening to explore the strategies used for screening and the key outcomes of screening programs including participation rates, testing rates, treatment rates and the positivity.

\section{Methods}

This systematic review was conducted according to the PRISMA guidelines [21].

\section{Search strategy}

The electronic bibliographic databases, PubMed and EMBASE, were searched for English language studies published between January 1, 2005 and January 28, 2011 with the search terms: Chlamydia, or Chlamydia infections, or Chlamydia trachomatis, OR Gonorrhea, AND Screening, or Mass Screening, or testing. The search was restricted to 2005 onwards, since most programs involving home-based screening have been established in recent years. The reference lists of selected studies were screened for other potentially relevant studies.

\section{Inclusion criteria}

Papers were reviewed by two authors independently and disagreements were resolved by discussion and consensus. A study was included if it described a CT or CT and NG screening program with self-collected specimens at home and reported the number of tests. For studies in more than one setting, only home-based screening data were included. RCTs were included, with the data from home-testing arm only.

Studies were excluded if: no original data was reported, such as reviews or editorials; screening was conducted in both clinics and home but home-screening data were not reported separately; screening was conducted as part of a cohort study as the testing rates would be falsely elevated; or screening was anonymous where the test results could not be provided back to individuals.

\section{Data extraction and analysis}

One author extracted the data from each paper and a second author verified the data. The following information was extracted: demographics; recruitment strategy (target group, advertisement, reminders); specimens collected; test-kit and specimen delivery method; incentives provided; number of people invited, participated and screened; CT/NG positivity; notification of results and treatment; and the cost of tests (also converted to US dollars for comparison). The authors were contacted to collect additional information, if required.

Quantitative outcomes, either extracted or manually calculated from the raw data, were:

- Participation rate: Number of participants divided by number of individuals invited $\times 100$

- Specimen return rate: Number of specimens divided by number of participants $\times 100$

- Testing rate: Number of specimens (number of tests if not reported) divided by number of individuals invited $\times 100$

- CT/NG positivity: Number of positive tests divided by total tests $\times 100$

- Treatment rate: Number of individuals treated divided by number of positive tests $\times 100$

'Participants' were defined as individuals who agreed to receive home-collection kits or postal test kits (PTKs) 
on invitation, requested PTKs through the internet or phone, picked-up PTKs from designated locations, or completed a questionnaire in screening program.

Programs were classified into seven groups (hereafter called program type) based on the recruitment strategy, test-kit and specimen delivery method (Table 1). Programs were defined as population-based if participants were randomly selected from listing of all the individuals (or households) in the target population (e.g. voter register, telephone directory). For the studies presenting weighted CT/NG prevalence estimates, the crude positivity was calculated instead if the required data were available. A frequency analysis was conducted for all the variables. The median, inter-quartile range (IQR) and 95\% confidence intervals (CI) were calculated for the rates.

All the analyses were conducted in STATA 12 (StataCorp, College Station, TX, USA).

\section{Results}

The initial search led to 3219 unique papers, for which the titles and abstracts were screened (Figure 1). Fulltext manuscripts were reviewed for 259 papers, of which 221 were excluded. Of 38 selected, a further nine papers were excluded as they described the same programs as other papers [22-30], but any additional methodology information was extracted. No additional papers were identified from the reference lists.

A total of 29 papers were included in the review [31-59]. One paper described a program established in different settings in two phases [57], the outcomes are reported separately for both phases due to difference in the strategies used. For RCTs with more than one hometesting arm, the data from each arm is presented under the relevant program type $[40,43]$. The authors of four papers were contacted to collect additional information $[41,42,54,58]$.

\section{Overview of programs}

Programs involved outreach $(n=7)$, PTKs sent on invitation acceptance $(n=7)$, PTKs sent along with invitations $(n=5)$, PTKs requested over the internet or phone without invitations $(\mathrm{n}=4)$, PTKs offered by in-person invites $(n=4)$, PTKs picked-up from designated locations $(n=3)$ and the use of two or more of these strategies $(n=2)$ (Table 1). Programs were located in Europe (48\%), US (24\%), Australia/New Zealand (17\%) and other countries (10\%). Most programs (69\%) targeted both males and females. The specimens consisted of urine only (66\%), vaginal swab only (3\%), urine for men and vaginal/vulval swab or vaginal flush sample for women (31\%).

Across all programs, 81633 tests were conducted (median:550 per program). The overall median participation rate was $68.9 \%(\mathrm{n}=12)$ and median specimen return rate was $51.4 \%(\mathrm{n}=26)$. The highest median specimen return rate was in outreach programs $(96.5 \%)$, followed by programs providing PTKs on invitation acceptance (78.9\%), PTKs sent along with invitations (32.9\%), PTKs requested without invitation (31.8\%), PTKs offered inperson (21.4\%), and PTKs picked-up at designated locations (18.6\%). The overall median testing rate was $28.8 \%(n=19)$, with a CT positivity of $3.6 \%(n=27)$ and NG positivity of $0.8 \%(n=7)$ (Table 2). Eleven studies reported the treatment rate, with a median of $96 \%$ (range: 67-100\%).

\section{Findings by program type \\ Outreach}

There were seven programs with participant recruitment in homes and immediate collection of specimens. Programs were conducted in the US [31,34,35], UK [32],

Table 1 Description of program type and specimen collection method

\begin{tabular}{|c|c|c|c|}
\hline Program type & Description & $\begin{array}{l}\text { Test-kit } \\
\text { delivery }\end{array}$ & $\begin{array}{l}\text { Specimen } \\
\text { return }\end{array}$ \\
\hline Outreach programs & Field staff recruited participants at their homes and collected specimens & In-person & In-person \\
\hline $\begin{array}{l}\text { PTK on invitation } \\
\text { acceptance }\end{array}$ & $\begin{array}{c}\text { People were invited through phone calls and/or letters to receive PTKs, those who agreed } \\
\text { were sent a PTK }\end{array}$ & Post & Post \\
\hline $\begin{array}{l}\text { PTK along with } \\
\text { invitation }\end{array}$ & People were sent PTKs along with invitation letters & Post & Post \\
\hline $\begin{array}{l}\text { PTK with in-person } \\
\text { invitation }\end{array}$ & People were directly invited to receive a PTK by study personnel & $\begin{array}{l}\text { In-person or } \\
\text { post }\end{array}$ & Post \\
\hline $\begin{array}{l}\text { PTK without } \\
\text { invitation }\end{array}$ & $\begin{array}{c}\text { PTKs were requested through the internet or phone without any direct invitations. Various } \\
\text { advertisement strategies were used }\end{array}$ & Post & $\begin{array}{l}\text { Post, drop- } \\
\text { off }\end{array}$ \\
\hline PTK with pick-up & $\begin{array}{l}\text { PTKs were picked-up from designated locations (e.g. from boxes at workplace) without } \\
\text { direct invitations }\end{array}$ & Pick-up & Post \\
\hline Multiple strategies & $\begin{array}{l}\text { A combination of different strategies was used, but outcomes were not presented } \\
\text { separately for each strategy }\end{array}$ & $\begin{array}{l}\text { In-person, post } \\
\text { or pick-up }\end{array}$ & Post \\
\hline
\end{tabular}

PTK, postal test kit. 


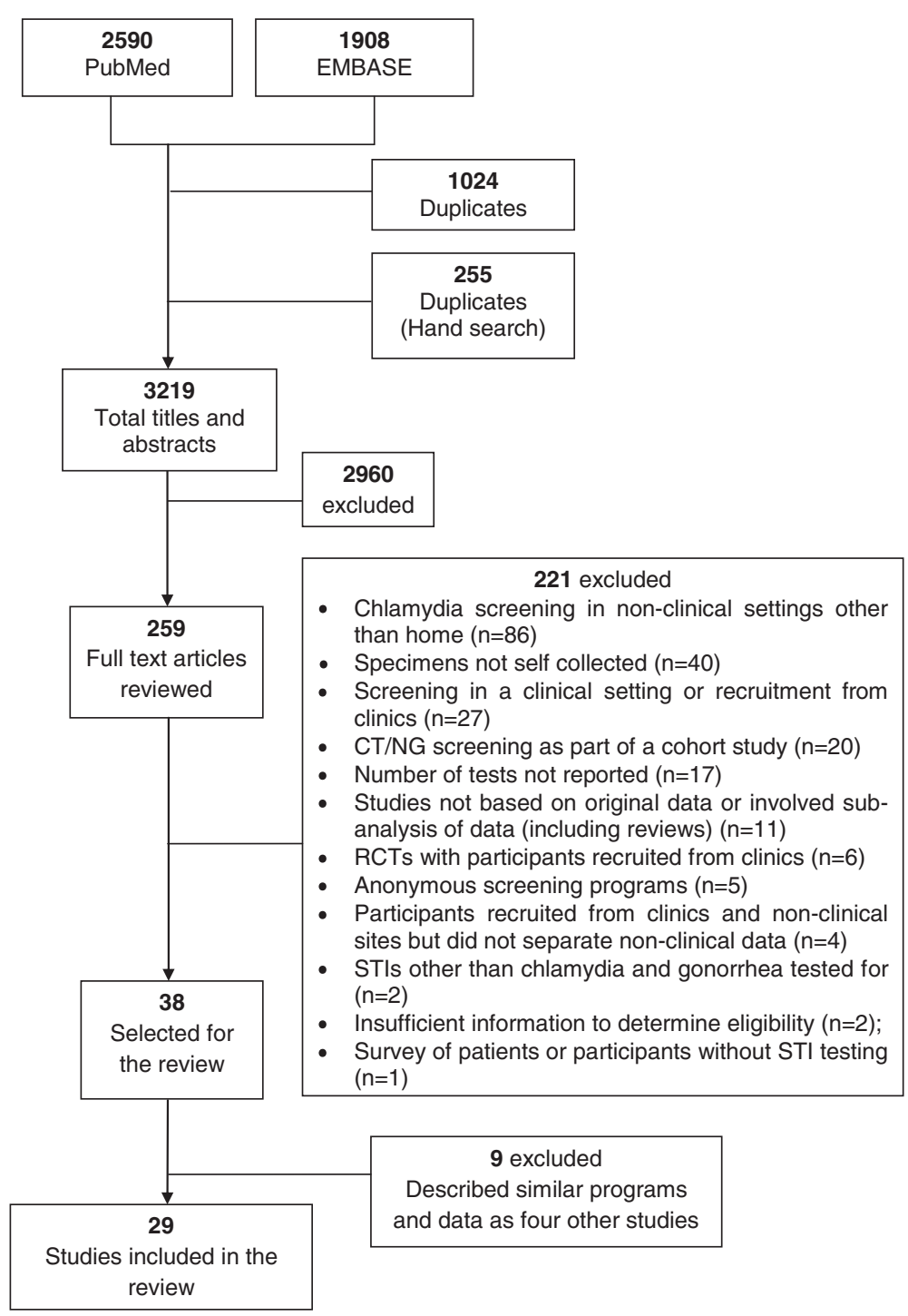

Figure 1 Flow diagram of systematic search strategy.

Barbados [36], Tanzania [33], Pakistan [37], and all were population-based (Table 3).

Across these programs, 13717 tests were conducted (median: 793). The median participation rate was $83.0 \%$ $(n=5)$, with a specimen return rate of $96.5 \%(n=6)$ and testing rate of $76.1 \%(n=5)$. The median CT positivity was $2.0 \%(n=5)$ and NG positivity was $0.7 \% \quad(n=4)$ (Table 2). Four programs encouraged participants to contact the staff for test results [31-34]. One program reported a $67 \%$ treatment rate $(n=49)$, with 24 of 28 traced sexual contacts (86\%) also treated [32].

\section{PTK on invitation acceptance}

In these seven programs, people were invited to receive PTKs through phone calls $(n=1)$, letters $(n=4)$, or letters and phone calls $(n=2)$. Programs were conducted in the
US [43,44], Australia [41], France [39], Denmark [40], Sweden [42] and the Netherlands [38] (Table 3). Six population-based programs randomly selected participants from population, health service or student registers $[38,40,42]$, telephone directories $[41,44]$, and from a national survey [39]. Another study was conducted at a health care plan [43]. In one program, participants requested PTKs through a website after receiving an invitation letter [38]. In this program, in less prevalent areas, only individuals with risk-scores above a certain level could request PTKs after mandatory online riskassessment [38]. In other programs, people requested a PTK via phone or by returning prepaid reply cards.

Across these programs, 46225 tests were conducted (median: 657). The median participation rate was $37.1 \%$ $(n=4)$, specimen return rate was $78.9 \%(n=5)$ and testing 
Table 2 Summary of home-based screening outcomes by program type

\begin{tabular}{|c|c|c|c|c|c|}
\hline Program type & $\begin{array}{c}\text { Tests } \\
\text { Total, median, IQR }\end{array}$ & $\begin{array}{l}\text { Participation rate } \\
\text { Median, IQR }\end{array}$ & $\begin{array}{c}\text { Specimen return rate } \\
\text { Median, IQR }\end{array}$ & $\begin{array}{l}\text { Testing rate } \\
\text { Median, IQR }\end{array}$ & $\begin{array}{l}\text { CT positivity } \\
\text { Median, IQR }\end{array}$ \\
\hline \multirow[t]{2}{*}{ Outreach programs } & $13717,793,402-3608$ & $83.0 \%, 82.3-87.8$ & $96.5 \%, 91.7-99.4$ & $76.1 \%, 70.7-82.3$ & $2.0 \%, 1.5-3.6$ \\
\hline & $(n=7)$ & $(n=5)$ & $(n=6)$ & $(n=5)$ & $(n=5)$ \\
\hline \multirow[t]{2}{*}{ PTK on invitation acceptance } & $46225,657,105-2580$ & $37.1 \%, 17.3-65.1$ & $78.9 \%, 68.3-86.0$ & $18.1 \%, 12.8-36.2$ & $2.0 \%, 1.0-4.2$ \\
\hline & $(n=7)$ & $(n=4)$ & $(n=5)$ & $(n=6)$ & $(n=7)$ \\
\hline \multirow[t]{2}{*}{ PTK along with invitation } & $15126,1296,486-4731$ & & $32.9 \%, 28.8-34.8$ & $28.8 \%, 23.9-28.8$ & $4.6 \%, 2.6-5.1$ \\
\hline & $(n=5)$ & & $(n=5)$ & $(n=5)$ & $(n=5)$ \\
\hline \multirow[t]{2}{*}{ PTK without invitation } & $2666,709,279-1055$ & & $31.8 \%, 26.5-47.4$ & & $9.1 \%, 5.2-12.8$ \\
\hline & $(n=4)$ & & $(n=4)$ & & $(n=3)$ \\
\hline \multirow[t]{2}{*}{ PTK with in-person invitation } & $341,37,5-166$ & $46.4 \%, 34.7-66.7$ & $21.4 \%, 19.7-87.5$ & $9.1 \%, 7.4-58.3$ & $1.5 \%, 0-9.1$ \\
\hline & $(n=4)$ & $(n=3)$ & $(n=3)$ & $(n=3)$ & $(n=3)$ \\
\hline \multirow[t]{2}{*}{ PTK with pick-up } & $1167,285,83-799$ & & $18.6 \%, 12.1-20.2$ & & $5.4 \%, 1.8-9.0$ \\
\hline & $(n=3)$ & & $(n=3)$ & & $(n=2)$ \\
\hline \multirow[t]{2}{*}{ Multiple strategies } & $2391,1196,96-2295$ & & & & $9.2 \%, 7.3-11.1$ \\
\hline & $(n=2)$ & & & & $(n=2)$ \\
\hline Overall & $81633,550,168-1368$ & $68.9 \%, 40.6-82.6$ & $51.4 \%, 22.0-87.5$ & $28.8 \%, 12.8-65.6$ & $3.6 \%, 1.7-7.3$ \\
\hline
\end{tabular}

CT, Chlamydia trachomatis; IQR, inter-quartile range; PTK, postal test kit.

rate was $18.8 \%(n=6)$. The median CT positivity was $2.0 \%(n=7)$ (Table 2). Reminders were used in four programs, which accounted for $41 \%$ of specimens in one program (1-5 phone calls, new PTK) [44], 39\% of specimens in the second program (two emails) [38] and an increased specimen return rate from $29 \%$ to $68 \%$ in the third program (phone call, two invitation letters, new PTK) [39]. The fourth program did not report the effect of reminders (one letter) [43].

Participants were either notified of test results $[39-41,43,44]$, or could access results online $[38,42]$. One program had a $100 \%$ treatment rate $(n=1)$ [43], a second program reported $84 \%$ treatment rate $(n=36)$ with $81 \%$ of contacts $(n=22)$ also treated [39], while a third program reported $89 \%$ treatment rate $(n=892)$ among $43 \%$ of positive individuals who completed a questionnaire [38]. The cost of one telephone survey and test-kit delivery was US\$250-300 in one program [44], and the cost of diagnosing one infection was SEK14000 (US\$2020) in another program [42].

\section{PTK sent along with invitation}

In these five programs, PTKs were sent to participants' homes along with invitation letters. Programs were conducted in the US [43], England [46], Denmark [40], Estonia [47] and the Netherlands [45] (Table 3). Four population-based programs randomly selected participants from the population register $[45,47]$, health service register [40] and general practice lists [46], while another program selected participants from a healthcare plan [43].
A total of 15126 tests were conducted (median: 4731). The median specimen return rate was $32.9 \%(\mathrm{n}=5)$ and testing rate was $28.8 \%(\mathrm{n}=5)$ (Table 2$)$. The median CT positivity was $4.6 \%(n=3)$ (Table 2$)$. Three programs used reminders, which accounted for $10 \%$ of specimens in one program (letter, phone call, home visit/flagging patient records) [46], 18\% of specimens in the second program (letter or new PTK) [45], while the third program did not report the effect of reminders [43].

Test results were notified to participants in all the programs. The treatment rate was reported in two programs, $100 \%(n=6)$ in one program [43] and $91 \%$ $(n=150)$ in the second program with $49 \%$ of partners $(n=86)$ also treated [45]. The operational cost of one program was $£ 14.65$ (US\$23) per invitation and $£ 21.47$ (US\$34) per individual screened [46].

\section{PTK without invitation}

These four programs in the US [48,50], Australia [51] and Sweden [49] used internet or telephone to request PTKs without any direct invitations (Table 3). These programs used several advertising strategies for promotion. The specimens were returned by post, except one program which required specimens to be dropped-off in boxes at selected locations [51]. In one program, in addition to the internet, PTKs could be picked-up at community locations but this method was discontinued due to a poor response rate [48].

A total of 2666 tests were conducted (median: 709) with the median specimen return rate of $31.8 \%(n=4)$. The median CT positivity was $9.1 \%(n=3)$ (Table 2$)$. Test 
Table 3 Strategies and outcomes of home-based CT and NG screening studies published between Jan 2005-Jan 2011 classified by program type

\begin{tabular}{|c|c|c|c|c|c|c|c|c|c|}
\hline Author, year & Country & Target group, recruitment & $\begin{array}{l}\text { Sex } \\
\text { Age }\end{array}$ & Tests & $\begin{array}{c}\text { Participation } \\
\text { rate \% } \\
(95 \% \mathrm{Cl})\end{array}$ & $\begin{array}{l}\text { Specimen } \\
\text { return } \\
\text { rate } \% \\
(95 \% \mathrm{Cl})\end{array}$ & $\begin{array}{l}\text { Testing } \\
\text { rate } \\
\% \\
(95 \% \mathrm{Cl})\end{array}$ & $\begin{array}{c}\text { CT } \\
\text { positive } \\
\% \\
(95 \% \mathrm{Cl})\end{array}$ & $\begin{array}{c}\text { NG } \\
\text { positive } \\
\% \\
(95 \% \mathrm{Cl})\end{array}$ \\
\hline \multicolumn{10}{|c|}{ Outreach Programs $(n=7)$} \\
\hline \multirow[t]{2}{*}{ Datta, 2007 [31] } & US & Screening within a national survey ${ }^{A}$ & $M / F$ & 6632 & 83.0 & 91.7 & 76.1 & $3.6 \%$ & $0.5 \%$ \\
\hline & & & $14-39$ & & $(82.2-83.8)$ & $(91.1-92.4)$ & $(75.2-77.0)$ & $(3.2-4.1)$ & $(0.4-0.8)$ \\
\hline \multirow{2}{*}{$\begin{array}{l}\text { McCadden, } \\
2005 \text { [32] }\end{array}$} & Brittain & Randomly selected (national survey ${ }^{\mathrm{B}}$ ) & $M / F$ & 3608 & 71.1 & 99.4 & 70.7 & $2.0 \%$ & \\
\hline & & & $18-44$ & & $(69.8-72.3)$ & $(99.1-99.7)$ & $(69.4-71.9)$ & $(1.6-2.5)$ & \\
\hline \multirow{2}{*}{$\begin{array}{l}\text { Ghebremichael, } \\
2009 \text { [33] }\end{array}$} & Tanzania & Randomly selected households & $\mathrm{F}$ & 1439 & 92.1 & 71.3 & 65.6 & $1.5 \%$ & $0.2 \%$ \\
\hline & & & $20-24$ & & $(90.9-93.2)$ & $(69.2-73.2)$ & $(63.6-67.6)$ & $(1.0-2.3)$ & $(0.0-0.6)$ \\
\hline \multirow{2}{*}{$\begin{array}{l}\text { Forhan, } 2009 \\
\text { [34] }\end{array}$} & US & Screening within a national survey ${ }^{A}$ & $\mathrm{~F}$ & 793 & & 94.6 & & $3.9^{C}$ & \\
\hline & & & $14-19$ & & & $(92.9-96.1)$ & & & \\
\hline \multirow{2}{*}{$\begin{array}{l}\text { Jennings, } 2010 \\
\text { [35] }\end{array}$} & US & Randomly selected households; Monetary & $M / F$ & 587 & 87.8 & 98.3 & 86.4 & & \\
\hline & & & $15-24$ & & $(85.1-90.2)$ & $(97.0-99.2)$ & $(83.6-88.9)$ & & \\
\hline \multirow{2}{*}{$\begin{array}{l}\text { Adams, } 2008 \\
{[36]}\end{array}$} & Barbados & Randomly selected (voter's register) & $\mathrm{M} / \mathrm{F}$ & $402^{\mathrm{D}}$ & $82.3 \%$ & 100 & 82.3 & $11.3 \%$ & $1.8 \%$ \\
\hline & & & $18-35$ & & $(78.6-85.5)$ & $(99.1-100)$ & $(78.6-85.5)$ & $(8.4-14.9)$ & $(0.7-3.6)$ \\
\hline \multirow[t]{2}{*}{ Mir, 2009 [37] } & Pakistan & Randomly selected households in a survey & $\mathrm{M}$; & 256 & & & & $0.0 \%$ & $0.8 \%$ \\
\hline & & & $16-45$ & & & & & & $(0.1-2.8)$ \\
\hline \multicolumn{10}{|c|}{ Programs with PTKs sent on invitation acceptance $(n=7)$} \\
\hline \multirow{2}{*}{$\begin{array}{l}\text { *Van Bergen, } \\
2010[38]\end{array}$} & Nether- & Participants form population register, PTKs & $M / F$ & 41638 & 20.2 & 78.9 & 16.0 & $4.2 \%$ & \\
\hline & & rec & $16-29$ & & $(20.1-20.4)$ & $(78.6-79.3)$ & $(15.8-16.1)$ & $(4.0-4.4)$ & \\
\hline \multirow{2}{*}{$\begin{array}{l}\text { Goulet, } 2010 \\
\text { [39] }\end{array}$} & France & nal survey); & $M / F$ & 2580 & 76.3 & 68.3 & 52.0 & $1.7 \%$ & \\
\hline & & & $18-44$ & & $(75.0-77.4)$ & $(66.7-69.7)$ & $(50.6-53.4)$ & $(1.2-2.2)$ & \\
\hline \multirow{2}{*}{$\begin{array}{l}\text { *Anderson, } \\
2010[40]\end{array}$} & Denmark & Randomly selected (county health service & $M / F$ & 912 & & & 20.3 & $7.0 \%$ & \\
\hline & & & $22-24$ & & & & $(19.1-21.5)$ & $(5.4-8.9)$ & \\
\hline \multirow{2}{*}{$\begin{array}{l}\text { Hocking, } 2006 \\
{[41]}\end{array}$} & Australia & Random household sample (telephone & $\mathrm{F}$; & 657 & $53.9^{\mathrm{E}}$ & 67.1 & $36.2^{\mathrm{E}}$ & $0.9 \%$ & \\
\hline & & & $18-35$ & & $(51.6-56.2)$ & $(64.1-70.0)$ & $(33.9-38.4)$ & $(0.3-2.0)$ & \\
\hline \multirow{2}{*}{$\begin{array}{l}\text { Domeika, } 2007 \\
\text { [42] }\end{array}$} & Sweden & Randomly selected (population register, & $M / F$ & 247 & 14.5 & 88.2 & 12.8 & $2.0 \%$ & \\
\hline & & & $19-23$ & & $(12.9-16.1)$ & $(83.8-91.7)$ & $(11.3-14.3)$ & $(0.7-4.7)$ & \\
\hline \multirow{2}{*}{$\begin{array}{l}\text { *Scholes, } 2007 \\
{[43]}\end{array}$} & US & Participants from enrollees in a managed & M; & 105 & & & 3.6 & $1.0 \%$ & \\
\hline & & & $21-25$ & & & & $(2.9-4.3)$ & $(0.0-5.2)$ & \\
\hline \multirow{2}{*}{$\begin{array}{l}\text { Eggleston, } 2005 \\
{[44]}\end{array}$} & US & Telephone accessible households; & $M / F$ & 86 & & 86.0 & & $2.3 \%$ & $0.0 \%$ \\
\hline & & & $18-35$ & & & $(77.6-92.1)$ & & $(0.3-8.1)$ & \\
\hline \multicolumn{10}{|c|}{ Programs with PTKs sent along with invitation $(n=5)$} \\
\hline \multirow{2}{*}{$\begin{array}{l}\text { Van Bergen, } \\
2005 \text { [45] }\end{array}$} & Nether- & Randomly selected (civilian registry); & $M / F$ & 8383 & & $40.3^{* *}$ & 39.9 & $2.0 \%$ & \\
\hline & & & $15-29$ & & & $(39.7-41.0)$ & $(39.3-40.6)$ & $(1.7-2.3)$ & \\
\hline \multirow[t]{2}{*}{ Low, 2007 [46] } & England & Randomly selected (general practice lists); & $M / F$ & 4731 & & $32.9^{* *}$ & 23.9 & $4.6 \%$ & \\
\hline & & & $16-39$ & & & $(32.1-33.7)$ & $(23.3-24.5)$ & $(4.0-5.3)$ & \\
\hline \multirow{2}{*}{$\begin{array}{l}{ }^{*} \text { Anderson, } \\
2010 \text { [40] }\end{array}$} & Denmark & ly selected (county health service & $M / F$ & 1296 & & 28.8 & 28.8 & $6.2 \%$ & \\
\hline & & & $22-24$ & & & $(27.5-30.1)$ & $(27.5-30.1)$ & $(4.9-7.6)$ & \\
\hline \multirow{2}{*}{$\begin{array}{l}\text { Uuskula, } 2008 \\
\text { [47] }\end{array}$} & Estonia & Randomly selected (population registry) & $M / F$ & 486 & & $34.8^{* *}$ & 28.8 & $5.1 \%$ & \\
\hline & & & $18-35$ & & & $(32.3-37.4)$ & $(26.7-31.0)$ & $(3.4-7.5)$ & \\
\hline \multirow{2}{*}{$\begin{array}{l}\text { *Scholes, } 2007 \\
{[43]}\end{array}$} & US & Participants from enrollees in a managed & M; & 230 & & $7.8(6.9-8.9)$ & 7.8 & $2.6 \%$ & \\
\hline & & & $21-25$ & & & & $(6.9-8.9)$ & $(1.0-5.6)$ & \\
\hline
\end{tabular}


Table 3 Strategies and outcomes of home-based CT and NG screening studies published between Jan 2005-Jan 2011 classified by program type (Continued)

PTKs without invitation programs $(n=4)$

\begin{tabular}{|c|c|c|c|c|c|c|c|c|}
\hline \multirow{2}{*}{$\begin{array}{l}\text { Gaydos, } 2009 \\
{[48]}\end{array}$} & \multirow[t]{2}{*}{ US } & \multirow{2}{*}{$\begin{array}{l}\text { PTKs requested through the internet; } \\
\text { Advertised }\end{array}$} & $\mathrm{F}$; & \multirow[t]{2}{*}{1203} & & 32.4 & & $9.1 \%$ \\
\hline & & & $>=14$ & & & $(30.9-33.9)$ & & $(7.5-10.8)$ \\
\hline \multirow{2}{*}{$\begin{array}{l}\text { Novak, } 2006 \\
{[49]}\end{array}$} & \multirow[t]{2}{*}{ Sweden } & \multirow{2}{*}{$\begin{array}{l}\text { PTKs requested through the internet; } \\
\text { Advertised }\end{array}$} & $\mathrm{M} / \mathrm{F}$ & \multirow{2}{*}{\multicolumn{2}{|c|}{906}} & 62.5 & & $5.2 \%$ \\
\hline & & & & & & $(59.9-65.0)$ & & $(3.8-6.8)$ \\
\hline \multirow[t]{2}{*}{ Chai, 2010 [50] } & \multirow[t]{2}{*}{ US } & \multirow{2}{*}{$\begin{array}{l}\text { PTKs requested through the internet; } \\
\text { Advertised }\end{array}$} & M; & \multirow{2}{*}{\multicolumn{2}{|c|}{512}} & 31.1 & & 12.8 \\
\hline & & & $>=14$ & & & $(28.9-33.4)$ & & $\begin{array}{l}(10.0- \\
16.0)\end{array}$ \\
\hline \multirow{3}{*}{$\begin{array}{l}\text { Martin, } 2009 \\
{[51]}\end{array}$} & \multirow[t]{2}{*}{ Australia } & \multirow{2}{*}{$\begin{array}{l}\text { PTKs requested through the internet/ } \\
\text { phone, specimens dropped-off; Advertised }\end{array}$} & $M / F$ & \multirow{2}{*}{\multicolumn{2}{|c|}{45}} & 22.0 & & \\
\hline & & & $16-24$ & & & $(16.5-28.2)$ & & \\
\hline & \multicolumn{8}{|c|}{ PTKs with in-person invitation programs $(n=4)$} \\
\hline \multirow{2}{*}{$\begin{array}{l}\text { Brabin, } 2009 \\
{[52]}\end{array}$} & \multirow[t]{2}{*}{ England } & \multirow{2}{*}{$\begin{array}{l}\text { PTKs offered to women requesting EHC at } \\
\text { pharmacies }\end{array}$} & $\mathrm{F}$ & \multirow[t]{2}{*}{264} & 46.4 & 19.7 & 9.1 & $9.1 \%$ \\
\hline & & & $<=24$ & & $(44.6-48.3)$ & $(17.6-21.9)$ & $(8.1-10.2)$ & $(5.9-13.2)$ \\
\hline \multirow{2}{*}{$\begin{array}{l}\text { Sacks-Davis, } \\
2010 \text { [53] }\end{array}$} & \multirow[t]{2}{*}{ Australia } & \multirow{2}{*}{$\begin{array}{l}\text { People at a music festival invited to } \\
\text { receive PTKs; Non-monetary incentive; } \\
\text { Reminders }\end{array}$} & $M / F$ & \multirow[t]{2}{*}{67} & 34.7 & 21.4 & 7.4 & $1.5 \%$ \\
\hline & & & $16-29$ & & $(31.6-37.9)$ & $(17.0-26.4)$ & $(5.4-9.3)$ & $(0.0-8.0)$ \\
\hline \multirow{2}{*}{$\begin{array}{l}\text { Dabrera, } 2010 \\
{[54]}\end{array}$} & \multirow[t]{2}{*}{ England } & \multirow{2}{*}{$\begin{array}{l}\text { PTKs offered to women requesting EHC at } \\
\text { pharmacies }\end{array}$} & $\mathrm{F}$; & \multirow[t]{2}{*}{7} & 66.7 & 87.5 & 58.3 & \\
\hline & & & $<=21$ & & $(34.9-90.1)$ & $(47.3-99.7)$ & $(27.7-84.8)$ & \\
\hline \multirow[t]{2}{*}{ Rose, 2010 [55] } & $\begin{array}{l}\text { New } \\
\text { Zealand }\end{array}$ & $\begin{array}{l}\text { PTKs offered to general practice clients to } \\
\text { pass to their social contacts }\end{array}$ & $M / F$ & \multicolumn{2}{|l|}{3} & & & $0.0 \%$ \\
\hline & & PTKs with pick- & -up pro & grams & $=3)$ & & & \\
\hline Davison, 2007 & Scotland & PTKs picked-up from GUM clinic, youth & $M / F$ & 799 & & 20.2 & & $9.0 \%$ \\
\hline & & & & & & $(18.9-21.5)$ & & $(7.1-11.2)$ \\
\hline MHF, 2005 [57] & England & PTKs (pick-up) were available to & $M_{i}$ & $285^{\mathrm{F}}$ & & 12.1 & & $1.8 \%$ \\
\hline & & Advertised & $<=30$ & & & $(10.8-13.5)$ & & $(0.6-4.0)$ \\
\hline MHF, 2005 [57] & England & PTKs available for pick-up at 5 non-clinical & $M_{i}$ & 83 & & $18.6^{\mathrm{G}}$ & & \\
\hline & & s & $<=30$ & & & $(15.1-22.5)$ & & \\
\hline & & Programs with mu & ultiple s & trateg & $(n=2)$ & & & \\
\hline Williamson, & Scotland & PTKs distributed or picked-up at various & $M / F$ & $2295^{\mathrm{H}}$ & & & & $11.1 \%$ \\
\hline & & & $13-25$ & & & & & $(9.9-12.5)$ \\
\hline Buhrer-Skinner, & Australia & PTKs requested through internet/phone or & M/F; & 100 & & & & $7.3 \%$ \\
\hline & & $\begin{array}{l}\text { picked-up at different locations; } \\
\text { Advertised }\end{array}$ & $16-25$ & & & & & $(3.0-14.4)$ \\
\hline
\end{tabular}

Definitions and abbreviations: Participation rate, participants divided by number invited $\times 100$; Specimen return rate, number of specimens (or tests) divided by participants $\times 100$; Testing rate, number of specimens divided by number invited $\times 100$. CT, Chlamydia trachomatis; NG, Neisseria gonorrhoeae; $\mathbf{M}$, Male; F, Female; US, United States; UK, United Kingdom; PTK, postal test kit; GUM, genitourinary medicine; MHF, Men's Health Forum.

* Randomized Controlled Trial (RCT) ** calculated among those who received PTKs (excluded undelivered kits).

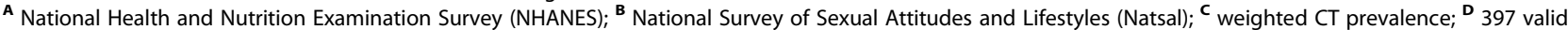
tests; ${ }^{\mathbf{E}}$ calculated among 1817 eligible contactable participants after excluding 6555 ineligible and 2629 un-contactable out of 11001 households sampled; ${ }^{\mathbf{F}}$ although the program was targeted at male employees, some of the specimens were returned by female employees; ${ }^{\mathbf{G}}$ specimen return rates for individual locations: Agricultural college, 41.0\% (41 tests); Factory, 36.0\% (9); Satellite college of university, 14.3\% (4); Military Police training center 13.6\% (12); Post-16 college $8.3 \%(17) ;{ }^{\mathbf{H}} 20 \%$ of returned kits were distributed from clinics and $10 \%$ were picked-up form university. 
results were accessible to participants in all the programs. The treatment rate in three programs was $97 \%$ $(n=105)$ [48], 99\% $(n=105)$ [50] and 100\% $(n=47)$ [49].

\section{PTK with in-person invitation}

Four programs involved study personnel directly inviting people to receive PTKs and were based in England [52,54], Australia [53] and New Zealand [55] (Table 3). Two programs were in pharmacies $[52,54]$, one in general practice [55] and one at a music festival [53]. A total of 341 tests were conducted (median: 37). The median participation rate was $46.4 \%(n=3)$, specimen return rate was $21.4 \% \quad(n=3)$ and testing rate was 9.1\% $(\mathrm{n}=3)$. The median CT positivity was $1.5 \%(\mathrm{n}=3)$ (Table 2). SMS reminders were used in one program [53]. Test results were notified to participants in two programs [53,55], and one program reported a $92 \%$ treatment rate $(n=22)$ [52].

\section{PTK with pick-up}

In these three programs, PTKs were available for pickup from designated locations [56,57] (Table 3). In the Men and Chlamydia Project (M\&CP) [57], employees of six workplaces in England picked-up PTKs from boxes in gents' toilets, locker rooms and restrooms. The second program, an extension of M\&CP [57], included five non-clinical sites. In the third program in Scotland [56], PTKs were picked-up from the door of a genitourinary medicine clinic, a youth service, family planning and other sources.

Across these programs, 1167 tests were conducted (median: 285). The median specimen return rate was $18.6 \%(\mathrm{n}=3)$ and the median CT positivity was $5.4 \%$ $(n=2)$ (Table 2). Results were notified to participants in the $M \& C P$ only and the treatment rate was $100 \%(n=5)$ [57]. The cost per test, excluding operational cost, in $\mathrm{M} \& \mathrm{CP}$ was $£ 8.36$ (US\$13) and the cost per diagnosed infection was $£ 695$ (US\$1079).

\section{Multiple strategies}

Two programs used more than one strategy for screening, but did not report the outcomes separately (Tables 2, 3). In an Australian study [59], PTKs were picked-up from pharmacies, tertiary education facilities, community groups and sports clubs, or requested through the internet and telephone. Only first 100 kits were analyzed and the treatment rate was $100 \%(n=7)$ [59]. In a Scottish study [58], PTKs were picked-up from boxes at commercial venues including pharmacies, young peoples' drop-ins and record stores, or distributed at drop-in venues, with 2295 specimens submitted for screening.

\section{Discussion}

Home-based CT and NG screening programs have been conducted in many countries with a range of strategies for recruitment, test-kit delivery and specimen-collection. A number of programs were population-based, most used PTKs and some involved the use of internet for requesting test-kits. The overall median specimen return rate for programs included in this review was $51.4 \%$, the median testing rate was $28.8 \%$ and the median CT positivity was $3.6 \%$.

The key strength of this systematic review is the large number and range of home-based screening programs from a number of countries, which allowed examination of different strategies and outcomes. We used standardised definitions for the key outcomes to allow comparisons within and across program types. However, there are a few limitations. Firstly, we did not search the grey literature and thus may not have included other relevant unpublished programs. Secondly, we were unable to report the key outcomes for all programs, such as participation and testing rates, due to design of the programs or necessary data not being reported. Thirdly, any comparison of $\mathrm{CT} / \mathrm{NG}$ positivity across the programs is limited by different target populations studied and known prevalence in the underlying populations.

This review included a number of outreach programs. The high specimen return rates in these programs indicate that majority of people agreed to provide specimens when approached in their homes. In these programs, specimen-collection was integrated into ongoing national [31,32,34] and population-based surveys [33,35-37], and hence incurred no significant additional cost and human resources. The downside of this approach is that such surveys are often conducted infrequently and are therefore more suitable for estimating the prevalence than being a method for ongoing screening.

The use of mailed specimens appears to have increased in popularity in recent years as most programs in this review used PTKs for screening. The recruitment strategies in these programs have varied considerably. Programs with PTKs mailed alongside an invitation have been implemented in a few European countries, but the specimen return rates were low. Programs which sent PTKs on invitation acceptance, on the other hand, were associated with relatively higher specimen return rates. Some PTK programs required the test-kits to be collected or offered at specific physical locations. These were associated with relatively less people being tested as well as low specimen return rates.

A few PTK programs in the review required the test-kits to be requested through the internet. PTKs requested in this way appear to be a logistically feasible strategy for establishing large population-based screening programs, 
as demonstrated by the initial results of a trial in the Netherlands [38]. The three year results of this registerbased yearly CT screening program published recently demonstrate no significant decrease in $\mathrm{CT}$ positivity in the target population after three screening rounds, with the testing rates declining in each round [60]. However, among people who were screened in all three years, the positivity dropped from $5.9 \%$ to $2.9 \%$ [60]. The number of individuals screened in the first round $(n=41638)$ was greater than in any other program in the review [38]. The internet can be useful for selective screening of high-risk people through the completion of online risk-assessment questionnaires, as done in less CT prevalent areas in the Netherlands [38]. Other internet programs in the review did not involve direct invitations and relied on people actively seeking PTKs after programs were advertised [48-50]. Relatively few people were tested by this approach but CT positivity was higher, which may be due to people self-selecting on the basis of their risk. The internet also provides an opportunity to deliver test results online in a confidential manner at the individual's convenience $[38,42,49]$.

The use of reminders has shown to improve the specimen return rates [38,39,44-46]. However, reminder implementation on a large scale, along with notification of results and contact-tracing, can be resource intensive [61] and may require comprehensive registries. There may be other logistic challenges in establishing PTK programs, such as collection and transport of specimens. Clinical specimens must comply with international and national packaging requirements for transport [62]. The current three-layered packaging system for infectious substances often results in large packages (at least one surface with minimum $100 \times 100 \mathrm{~mm}$ dimension) [63], which may require delivery and collection at the postoffice or through a courier, and thus costly to transport [62]. However, a recently developed sponge-based urinecollection device called UriSwab (Copan Diagnostics, Inc.) holds a small amount of urine after being held in the urine flow or dipped in a specimen cup. UriSwab is easy to transport, has shown good performance in detecting CT/NG infections and can potentially facilitate the establishment of PTK programs [64]. Other considerations in home-based screening programs may include the issues of privacy and confidentiality, such as ensuring communication of results, treatment and contact tracing in a confidential manner and in-line with the individual's preference $[61,62]$.

Home-based testing can potentially reduce individual screening costs by avoiding clinic fees as well as the indirect costs, such as time off work and transportation [65]. However, there is no conclusive evidence of the cost-effectiveness of home-based screening over routine clinic-based screening. Four programs in this review provided cost information in association with homebased screening $[42,44,46,57]$. Only Low and colleagues reported full operational cost of a PTK screening program [46], while Domeika et al. compared the cost of home-based screening in their study with routine screening and reported the former to be about five times higher [42]. One RCT compared the cost of home and clinic-based screening and found that home-screening provided a cost-saving ( $\$ 25$ per test in home vs. $\$ 111$ in clinic after including direct and indirect costs) [65], but that the cost-saving was not seen when the results were restricted to asymptomatic tests. It is thus important for future programs to focus on estimating operational costs in relation to the screening outcomes to establish the cost-effectiveness of home-based screening.

\section{Conclusions}

This systematic review shows that home-based screening programs have been conducted in various countries and have utilised a variety of strategies. Home-based testing with self-collected specimens appears to be an acceptable and logistically feasible method for CT and NG screening outside of clinics. However, economic evaluation of large-scale home screening programs is warranted to assess their cost-effectiveness in the real-world scenario. The recruitment and specimencollection strategies adopted for home-based screening and their potential impact on the outcomes need to be carefully considered. A pilot program to assess the feasibility of screening in the local context would be highly recommended before embarking on a largescale program. Ongoing assessment of the outcomes and subsequent modification of strategies should be considered to improve the effectiveness of screening programs.

\section{Abbreviations}

CT: Chlamydia trachomatis; NG: Neisseria gonorrhoeae; NAAT: Nucleic acid amplification test; PTK: Postal test kit; STI: Sexually transmissible infection; US: United States; UK: United Kingdom; RCT: Randomized controlled trial; Cl: Confidence interval; IQR: Inter-quartile range; M\&CP: Men and Chlamydia Project.

\section{Competing interests}

The authors declare that they have no competing interests. No funding was received by the authors for this systematic review.

\section{Authors' contributions}

RJG and MSJ conceptualized the study and developed the search strategy. MSJ conducted the search. MSJ and RJG reviewed the papers with input from HMB, JSH and JW and extracted the data. HW performed the statistical analysis. MSJ and RJG drafted the manuscript. All the authors read and approved the final manuscript.

\section{Author details}

${ }^{1}$ The Kirby Institute, University of New South Wales, Sydney, NSW, Australia. ${ }^{2}$ Center for Women's Health, Gender and Society, Melbourne School of Population Health, University of Melbourne, Melbourne, VIC, Australia. ${ }^{3}$ Division of Epidemiology, School of Public Health, University of California, Berkeley, CA, USA. ${ }^{4}$ Centre for Excellence in Rural Sexual Health, Rural Health 
Academic Centre, Melbourne Medical School, University of Melbourne, Melbourne, VIC, Australia. ${ }^{5}$ Sydney Sexual Health Center, Sydney Hospital, Sydney, NSW, Australia.

\section{Received: 23 August 2012 Accepted: 27 February 2013}

Published: 4 March 2013

\section{References}

1. Centers for Disease Control and Prevention: Sexually transmitted disease surveillance 2010. Atlanta: U.S. Department of Health and Human Services; 2011.

2. European Centre for Disease Prevention and Control: Sexually transmitted infections in Europe 1990-2010. Stockholm: ECDC; 2010.

3. NNDSS Annual Report Writing Group: Australia's notifiable disease status, 2010: annual report of the National Notifiable Disease Surveillance System. Commun Dis Intell 2012, 36(1):1-69.

4. Hook E III, Handsfield HH: Gonococcal infections in the adult. In Sexually Transmitted Diseases. 3rd edition. Edited by Holmes K, Mardh P, Sparling P, et al. New York, NY: McGraw-Hill; 1999.

5. Peipert JF: Genital chlamydial infections. N Engl J Med 2003, 349(25):2424-2430

6. Harris M, Bennett J, Del Mar C, Fasher M, Foreman L, Furler J, Johnson C, Joyner B, Litt J, Mazza D, Smith J, Tomlins R, London J, Snowdon T: Guidelines for preventive activities in general practice (the 'Red Book'). 7th edition. Melbourne: The Royal Australian College of General Practitioners; 2009.

7. Lanjouw E, Ossewaarde JM, Stary A, Boag F, van der Meijden WI: European guideline for the management of Chlamydia trachomatis infections. Int J STD AIDS 2010, 21(11):729-737.

8. Workowski KA, Berman S: Sexually transmitted diseases treatment guidelines, 2010. MMWR Recomm Rep 2010, 59(RR-12):1-110.

9. European Centre for Disease Prevention and Control: Chlamydia control in Europe. Stockholm: ECDC; 2009.

10. Kong F, Guy RJ, Hocking JS, Merritt T, Pirotta M, Heal C, Bergeri I, Donovan B, Hellard ME: Australian general practitioner chlamydia testing rates among young people. Med J Aust 2011, 194(5):249-252.

11. Levine WC, Dicker LW, Devine O, Mosure DJ: Indirect estimation of chlamydia screening coverage using public health surveillance data. Am J Epidemiol 2004, 160(1):91-96.

12. St. Lawrence JS, Montaño DE, Kasprzyk D, Phillips WR, Armstrong K, Leichliter JS: STD screening, testing, case reporting, and clinical and partner notification practices: a national survey of US physicians. Am J Public Health 2002, 92(11):1784-1788.

13. Hocking J, Parker R, Pavlin N, Fairley C, Gunn J: What needs to change to increase chlamydia screening in general practice in Australia? The views of general practitioners. BMC Publ Health 2008, 8(1):425.

14. McNulty CA, Elaine F, Jo B, Julia S, Kevin AF: Barriers to opportunistic chlamydia testing in primary care. Br J Gen Pract 2004, 54(504):508-514.

15. Booth ML, Bernard D, Quine S, Kang MS, Usherwood T, Alperstein G Bennett DL: Access to health care among Australian adolescents young people's perspectives and their sociodemographic distribution. J Adolesc Health 2004, 34(1):97-103.

16. Ford CA, Bearman PS, Moody J: Foregone health care among adolescents. JAMA 1999, 282(23):2227-2234.

17. Marcell AV, Klein JD, Fischer I, Allan MJ, Kokotailo PK: Male adolescent use of health care services: where are the boys? J Adolesc Health 2002, 30(1):35-43

18. Graseck AS, Shih SL, Peipert JF: Home versus clinic-based specimen collection for Chlamydia trachomatis and Neisseria gonorrhoeae. Expert Rev Anti Infect Ther 2011, 9(2):183-194.

19. Shih SL, Graseck AS, Secura GM, Peipert JF: Screening for sexually transmitted infections at home or in the clinic? Curr Opin Infect Dis 2011 24(1):78.

20. Graseck AS, Secura GM, Allsworth JE, Madden T, Peipert JF: Home compared with clinic-based screening for sexually transmitted infections: a randomized controlled trial. Obstet Gynecol 2010, 116(6):1311-1318

21. Ottawa Hospital Research Institute: PRISMA: Transparent Reporting of Systematic Reviews and Meta-Analyses. 2010. http://www.prisma-statement. org. (accessed 1 December).

22. Gotz HM, Veldhuijzen IK, Van Bergen JEAM, Hoebe CJPA, De Zwart O, Richardus JH, Broer J, Coenen AJJ, De Groot F, Van Schaik DT, Verhooren
MJC: Acceptability and consequences of screening for chlamydia trachomatis by home-based urine testing. Sex Transm Dis 2005, 32(9):557-562

23. Gaydos CA, Barnes M, Aumakhan B, Quinn N, Wright C, Agreda P, Whittle P, Hogan T: Chlamydia trachomatis age-specific prevalence in women who used an internet-based self-screening program compared to women who were screened in family planning clinics. Sex Transm Dis 2011, 38(2):74-78.

24. Gaydos CA, Dwyer K, Barnes M, Rizzo-Price PA, Wood BJ, Flemming T, Hogan MT: Internet-based screening for Chlamydia trachomatis to reach nonclinic populations with mailed self-administered vaginal swabs. Sex Transm Dis 2006, 33(7):451-457.

25. Gotz HM, Hoebe CJPA, Van Bergen JEAM, Veldhuijzen IK, Broer J, De Groot F, Verhooren MJC, Van Schaik DT, Coenen AJJ, Richardus JH: Management of Chlamydia cases and their partners: Results from a home-based screening program organized by Municipal Public Health Services with referral to regular health care. Sex Transm Dis 2005, 32(10):625-629.

26. Gotz HM, Van Bergen JEAM, Veldhuijzen IK, Hoebe CJPA, Broer J, Coenen AJJ, De Groot F, Verhooren MJC, Van Schaik DT, Richardus JH: Lessons learned from a population-based chlamydia screening pilot. Int J STD AIDS 2006, 17(12):826-830.

27. Macleod J, Salisbury C, Low N, McCarthy A, Sterne JAC, Holloway A, Patel R, Sanford E, Morcom A, Horner P, Smith GD, Skidmore S, Herring A, Caul O, Hobbs FDR, Egger M: Coverage and uptake of systematic postal screening for genital Chlamydia trachomatis and prevalence of infection in the United Kingdom general population: Cross sectional study. BMJ 2005, 330(7497):940-942.

28. Masek BJ, Arora N, Quinn N, Aumakhan B, Holden J, Hardick A, Agreda P, Barnes M, Gaydos CA: Performance of three nucleic acid amplification tests for detection of Chlamydia trachomatis and Neisseria gonorrhoeae by use of self-collected vaginal swabs obtained via an internet-based screening program. J Clin Microbiol 2009, 47(6):1663-1667.

29. Van Bergen J, Gotz H, Richardus JH, Hoebe C, Broer J, Coenen T: Prevalence of urogenital Chlamydia trachomatis infections in the Netherlands suggests selective screening approaches. Results from the pilot CT population study. Drugs Today (Barc) 2006, 42(SUPPL. A):25-33.

30. Van den Broek IV, Hoebe CJ, van Bergen JE, Brouwers EE, de Feijter EM, Fennema JS, Gotz HM, Koekenbier RH, van Ravesteijn SM, de Coul EL: Evaluation design of a systematic, selective, internet-based, chlamydia screening implementation in the Netherlands, 2008-2010: implications of first results for the analysis. BMC Infect Dis 2010, 10:89.

31. Datta SD, Sternberg M, Johnson RE, Berman S, Papp JR, McQuillan G, Weinstock H: Gonorrhea and chlamydia in the United States among persons 14 to 39 years of age, 1999 to 2002. Ann Intern Med 2007, 147(2):89-96.

32. McCadden A, Fenton KA, McManus S, Mercer CH, Erens B, Carder C, Ridgway G, MacDowall W, Nanchahal K, Byron CL, Coaps A, Wellings K, Johnson AM: Chlamydia trachomatis testing in the second British National Survey of sexual attitudes and lifestyles: Respondent uptake and treatment outcomes. Sex Transm Dis 2005, 32(6):387-394.

33. Ghebremichael M, Paintsil E, Larsen U: Alcohol abuse, sexual risk behaviors, and sexually transmitted infections in women in moshi Urban district, Northern Tanzania. Sex Transm Dis 2009, 36(2):102-107.

34. Forhan SE, Gottlieb SL, Sternberg MR, Xu F, Datta SD, McQuillan GM, Berman SM, Markowitz LE: Prevalence of sexually transmitted infections among female adolescents aged 14 to 19 in the United States. Pediatrics 2009, 124(6):1505-1512.

35. Jennings JM, Taylor R, lannacchione VG, Rogers SM, Chung SE, Huettner S, Ellen JM: The available pool of sex partners and risk for a current bacterial sexually transmitted infection. Ann Epidemiol 2010, 20(7):532-538.

36. Adams OP, Carter AO, Prussia P, McIntyre G, Branch SL: Risk behaviour, healthcare access and prevalence of infection with Chlamydia trachomatis and Neisseria gonorrhoeae in a population-based sample of adults in Barbados. Sex Transm Infect 2008, 84(3):192-194.

37. Mir AM, Wajid A, Reichenbach L, Khan M: STI prevalence and associated factors among urban men in Pakistan. Sex Transm Infect 2009, 85(3):199-200

38. Van Bergen JE, Fennema JS, Van den Broek IV, Brouwers EE, de Feijter EM, Hoebe CJ, Koekenbier RH, Op de Coul ELM, Van Ravesteijn SM, Gotz HM: Rationale, design, and results of the first screening round of a 
comprehensive, register-based, Chlamydia screening implementation programme in the Netherlands. BMC Infect Dis 2010, 10:293.

39. Goulet V, de Barbeyrac B, Raherison S, Prudhomme M, Semaille C, Warszawski J: Prevalence of Chlamydia trachomatis: results from the first national population-based survey in France. Sex Transm Infect 2010, 86(4):263-270

40. Andersen B, van Valkengoed I, Sokolowski I, Moller JK, Ostergaard L, Olesen F: Impact of intensified testing for urogenital Chlamydia trachomatis infections: a randomised study with 9-year follow-up. Sex Transm Infect 2010, 87:156-161.

41. Hocking JS, Willis J, Tabrizi S, Fairley CK, Garland SM, Hellard M: A chlamydia prevalence survey of young women living in Melbourne, Victoria. Sex Health 2006, 3(4):235-240

42. Domeika M, Oscarsson L, Hallen A, Hjelm E, Sylvan S: Mailed urine samples are not an effective screening approach for Chlamydia trachomatis case finding among young men. J Eur Acad Dermatol Venereol 2007, 21(6):789-794

43. Scholes D, Heidrich FE, Yarbro P, Lindenbaum JE, Marrazzo JM: Populationbased outreach for chlamydia screening in men: Results from a randomized trial. Sex Transm Dis 2007, 34(11):837-839.

44. Eggleston E, Turner CF, Rogers SM, Roman A, Miller WC, Villarroel MA, Ganapathi L: Monitoring STI prevalence using telephone surveys and mailed urine specimens: A pilot test. Sex Transm Infect 2005, 81(3):236-238.

45. Van Bergen J, Gotz HM, Richardus JH, Hoebe CJPA, Broer J, Coenen AJT: Prevalence of urogenital Chlamydia trachomatis increases significantly with level of urbanisation and suggests targeted screening approaches: Results from the first national population based study in the Netherlands. Sex Transm Infect 2005, 81(1):17-23.

46. Low N, McCarthy A, Macleod J, Salisbury C, Campbell R, Roberts TE, Horner P Skidmore S, Sterne JA, Sanford E, Ibrahim F, Holloway A, Patel R, Barton PM, Robinson SM, Mills N, Graham A, Herring A, Caul EO, Smith GD, Hobbs FDR Ross JDC, Egger M: Epidemiological, social, diagnostic and economic evaluation of population screening for genital chlamydial infection. Health Technol Assess 2007, 11(8):1-165.

47. Uuskula A, Kals M, Denks K, Nurm UK, Kasesalu L, DeHovitz J, McNutt LA: The prevalence of chlamydial infection in Estonia: A population-based survey. Int J STD AIDS 2008, 19(7):455-458.

48. Gaydos CA, Barnes M, Aumakhan B, Quinn N, Agreda P, Whittle P, Hogan T: Can E-technology through the internet be used as a new tool to address the chlamydia trachomatis epidemic by home sampling and vaginal swabs? Sex Transm Dis 2009, 36(9):577-580.

49. Novak DP, Karlsson RB: Simplifying chlamydia testing: An innovative Chlamydia trachomatis testing approach using the internet and a home sampling strategy: Population based study. Sex Transm Infect 2006 82(2):142-147

50. Chai SJ, Aumakhan B, Barnes M, Jett-Goheen M, Quinn N, Agreda P, Whittle P, Hogan T, Jenkins WD, Rietmeijer CA, Gaydos CA: Internet-based screening for sexually transmitted infections to reach nonclinic populations in the community: Risk factors for infection in men. Sex Transm Dis 2010, 37(12):756-763

51. Martin L, Freedman E, Burton L, Rutter S, Knight V, D'Amato A, Murray C, Drysdale J, Harvey S, McNulty A: The C-project: Use of self-collection kits to screen for Chlamydia trachomatis in young people in a communitybased health promotion project. Sex Health 2009, 6(2):157-162.

52. Brabin L, Thomas G, Hopkins M, O'Brien K, Roberts SA: Delivery of chlamydia screening to young women requesting emergency hormonal contraception at pharmacies in Manchester, UK: A prospective study. BMC Womens Health 2009, 9:7. doi:10.1186/1472-6874-9-7.

53. Sacks-Davis R, Gold J, Aitken CK, Hellard ME: Home-based chlamydia testing of young people attending a music festival - who will pee and post? BMC Publ Health 2010, 10:376.

54. Dabrera G, Pinson D, Whiteman S: Missed opportunities for chlamydia screening: An audit of community pharmacy activity. HIV Med 2010, 11:87-88.

55. Rose SB, Lawton BA, Bromhead C, MacDonald EJ, Elley CR: Poor uptake of self-sample collection kits for Chlamydia testing outside primary care. Aust N Z J Publ Health 2010, 34(5):517-520.

56. Davison R, Bartholemew S, Clutterbuck DJ: Audit of postal test kits in Edinburgh for Chlamydia trachomatis as an alternative to genitourinary medicine clinic attendance. Int J STD AIDS 2007, 18(5):349-350.
57. Men's Health Forum: Urine kits urge men to take chlamydia test. Pharm J 2005, 274(7343):384

58. Williamson LM, Scott G, Carrick-Anderson K, Kernaghan K, Hart GJ: Chlamydia trachomatis testing among 13-25-year-olds in nongenitourinary medicine settings. J Fam Plann Reprod Health Care 2007, 33(3):177-182.

59. Buhrer-Skinner M, Muller R, Bialasiewicz S, Sloots TP, Debattista J, Gordon R, Buettner PG: The check is in the mail: Piloting a novel approach to Chlamydia trachomatis testing using self-collected, mailed specimen. Sex Health 2009, 6(2):163-169.

60. Van den Broek IVF, Van Bergen JEAM, Brouwers EEHG, Fennema JSA, Götz HM, Hoebe CJPA, Koekenbier RH, Kretzschmar M, Over EAB, Schmid BV, Pars LL, Van Ravesteijn SM, Van der Sande MAB, de Wit GA, Low N, Op de Coul ELM: Effectiveness of yearly, register based screening for chlamydia in the Netherlands: controlled trial with randomised stepped wedge implementation. BMJ 2012, 345:e4316.

61. Ford CA, Viadro Cl, Miller WC: Testing for Chlamydial and Gonorrheal infections outside of clinic settings: A summary of the literature. Sex Transm Dis 2004, 31(1):38-51.

62. Bates SM, Rogstad KE: Postal research: too many problems? Sex Transm Infect 2000, 76(5):332-334.

63. World Health Organization: Guidance on regulations for the transport of infectious substances 2007-08. Geneva, Switzerland: WHO; 2007. http://www who.int/csr/resources/publications/biosafety/WHO_CDS_EPR_2007_2cc.pdf. (accessed January 20, 2012)

64. Castriciano S, Montrucchio G, Moglia R, Concialdi E, Oddone L, Bolla C, Biglino A: Comparison of Copan UriSwab with BD ProbeTec urine preservative transport kit for preservation and detection of CT and NG in the ProbeTec assay. Sex Transm Infect 2011, 87(Suppl 1):A277-A278.

65. Smith KJ, Cook RL, Ness RB: Cost comparisons between home-and clinicbased testing for sexually transmitted diseases in high-risk young women. Infect Dis Obstet Gynecol 2007, 2007:62467.

\section{doi:10.1186/1471-2458-13-189}

Cite this article as: Jamil et al:: Home-based chlamydia and gonorrhoea screening: a systematic review of strategies and outcomes. BMC Public Health 2013 13:189.

\section{Submit your next manuscript to BioMed Central and take full advantage of:}

- Convenient online submission

- Thorough peer review

- No space constraints or color figure charges

- Immediate publication on acceptance

- Inclusion in PubMed, CAS, Scopus and Google Scholar

- Research which is freely available for redistribution

Submit your manuscript at www.biomedcentral.com/submit
C BioMed Central 